\title{
Viewpoint
}

\section{Rush hour in a drop of coffee}

\section{Greg Huber}

Center for Cell Analysis \& Modeling and Department of Cell Biology, University of Connecticut Health Center, Farmington, CT 06030, USA and

Department of Physics and Department of Mathematics, University of Connecticut, Storrs, CT 06269,

$U S A$

Published August 15, 2011

The dramatic speed-up of fluid flow in a drying drop of a colloidal suspension controls the pattern of order and disorder in the particle stain that is left behind.

Subject Areas: Fluid Dynamics, Soft Matter

\author{
A Viewpoint on: \\ Order-to-Disorder Transition in Ring-Shaped Colloidal Stains \\ Álvaro G. Marín, Hanneke Gelderblom, Detlef Lohse, and Jacco H. Snoeijer \\ Phys. Rev. Lett. 107, 085502 (2011) - Published August 15, 2011
}

A drop of fluid dries, a pattern forms. Could anything be more mundane? Yet, as technological designs call for ever smaller devices, understanding how the flow of a particle-laden fluid could control the deposition of tiny objects is actually becoming a compelling intellectual challenge. In many applications, ranging from aggregating proteins to high-density information storage and photonics, the physics of how evaporating fluid can shape a pattern - the so-called coffee ring stain-looms large. Now, writing in Physical Review Letters, Álvaro Marín and colleagues at the University of Twente in the Netherlands report experiments in which they have tracked the structural pattern and dynamics of particles at the edge of the canonical ringlike stain [1].

Research on the coffee-ring phenomenon began with Sidney Nagel, a physics professor at the University of Chicago, who was curious about the form of coffee stains on his kitchen countertops 2]. Nagel's befuddlement, which he shared with his colleagues, could be stated this way: Why does all the material suspended in a drop of coffee end up at the edge when the drop evaporates, considering that it started out dispersed across the whole drop? The effect was common to all droplets of dispersed colloidal objects, including milk, blood, ink, and paint, evaporating on a wide variety of surfaces, suggesting there should be a general explanation.

The physical picture that emerged was beautiful and simple: As the droplet dries, the liquid evaporating from the thinning outer edge, where the contact angle $\theta$ is shrinking to zero, must be replenished by liquid from the drop's interior. This sets up a strong outward flow in the solvent, which carries most of the solute to the contact line. Pre-existing surface roughness can provide the force to pin the contact line, but the contact line further pins itself through a feedback loop between flow

DOI: $10.1103 /$ Physics.4.65

URL: http://link.aps.org/doi/10.1103/Physics .4.65 and patterning: the outward flow increases the deposition of solute, which serves to anchor the fluid and reinforce the outward flow.

The simplicity of this picture carries some caveats - the suppression of counterflows that are due to gradients in surface tension (Marangoni flows) is one example. However, in the years since Nagel's observation, the coffee ring has taken on a life of its own. Special-effects artists at the University of Washington and Pixar, for example, used the theory to improve their computer rendering of the luminous textures of watercolor paintings [3].

While the basic principles of the ringlike stain were first understood 15 years ago, a complete picture of how the deposition evolves in space and time have remained elusive. This is partly because we now appreciate how complicated the coffee-ring effect actually is: the contactline dynamics are subtle (such as depinning-repinning cycles) and researchers are discovering an expanding taxonomy of patterns (such as multiple rings, stripes, spikes, chevrons, and fractal-like lace) [4 6]. But even for the classic coffee ring, detailed microscopic information connecting structure to dynamics has simply not been available.

In their new experiments, Marín et al. follow the entire time evolution of a 3-microliter water droplet laden with micron-diameter fluorescent polystyrene beads sitting on a glass slide. Viewing the underside of the drop using an inverted microscope, they observe that there is a clear spatial dependence in the structure of the resulting ring as one moves in from the contact line toward the center of the droplet. Most dramatically, they see a structural transition from beads that are ordered, as in a crystal, to a more disordered packing arrangement (Fig. 11).

In order to pin down the origin of the patterning, the group tracked the particle velocities using micro-particle

(C) 2011 American Physical Society 


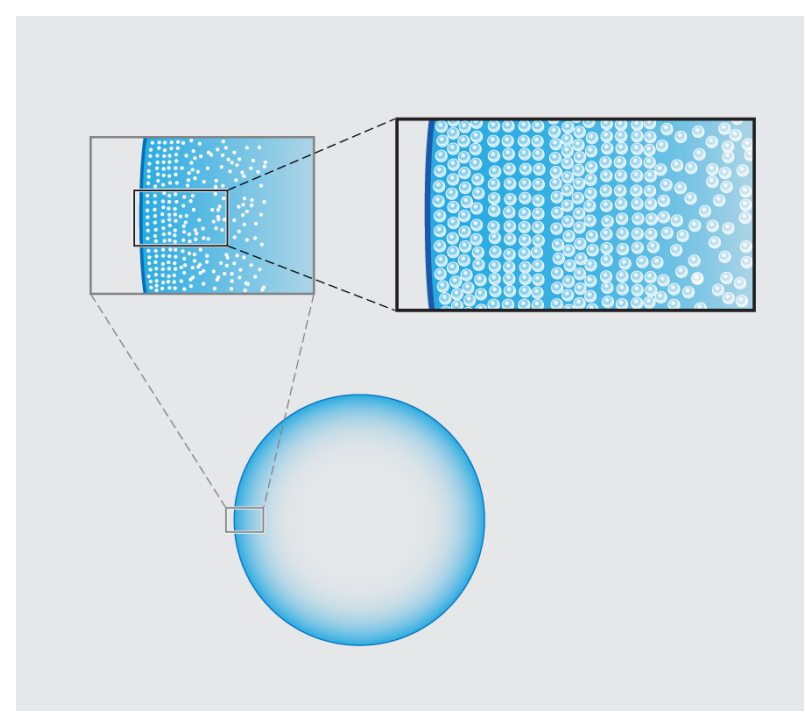

FIG. 1: An evaporating drop of liquid, laden with micron-size spherical particles, leaves a ringlike stain on the underlying surface. Light and electron microscopy reveal variations in the structure of the ring - crystalline near the outer edge and disordered toward the inner part - that occurs during evaporation. The high-zoom image in the upper right shows the succession of hexagonal and square packing arrangements characteristic of a layer of colloidal particles confined to a wedge. (APS/Alan Stonebraker)

image velocimetry ( $\mu \mathrm{PIV})$. Their basic result is quite simple: the spatial transition from ordered, crystalline arrays to disordered packings stems from a dramatic temporal speed up of the particle motion near the end of the evaporation, which the Twente team refer to as "the rush hour effect". The observation of this effect is not in itself new. A consequence of mass balance is that the height-averaged radial velocity $u$ at a distance $x$ from the droplet's edge behaves like $u \sim 1 /(\theta \sqrt{x})$. This implies both a spatial and temporal divergence of the particle velocity (as $x$ and $\theta$ approach zero, respectively) in the last stage of the droplet's life [2]. Marín et al.'s novel point is to argue that the particles forming the ordered phase arrive early at the contact line, when the deposition rate is low; those forming the disordered phase arrive during rush hour, at high deposition rates. The slow-to-arrive particles have the time to position themselves by diffusion into a snug arrangement, but those coming at rush hour have no time to find an optimal fit and are quenched into a jammed packing.

This Brownian version of particle ordering (which the authors liken to a game of Tetris) can be expressed as a comparison of time scales: on the one hand, the Einsteinrelation timescale $\tau=d^{2} / D$, where $d$ is the particle diameter, and $D$ the particle diffusivity; on the other hand, the hydrodynamic timescale $t=L / u$, where $L$ is the average distance between nearby particles (a function of particle concentration). The critical velocity when these two timescales coincide is then $u \sim L D / d^{2}$. Marín et al.'s $\mu$ PIV measurements of the velocity one particle diameter above the substrate for various particle sizes and concentrations enable them to experimentally determine the critical time and velocity for the onset of the orderdisorder transition. Indeed, they find that their experimental velocities closely match those given by the comparison of hydrodynamic and diffusive rates.

Direct confirmation of the three-dimensional structure of the various colloidal packings is still missing. Marín et al. present both light microscopy of the bottom layer and scanning electron micrograph (SEM) images. These show ordered phases that appear square and hexagonal (Fig. 1. top right). Both are, plausibly, hexagonal closepacked (hcp) lattices, since different planar patterns can appear depending on the orientation of the crystal symmetry axes with respect to the plane of the substrate. What controls these orientational transitions is the effect of the confinement of a small number of layers between the substrate and the liquid-vapor interface. In two dimensions, hexagonal packing is the most efficient way to pack spheres, and in an unconstrained, three-dimensional space, hcp is the most favorable. But in a confined wedge space, whenever a new layer of particles is deposited, the square packing is slightly denser. A similar situation occurs when colloids are confined between planar walls, or in a cylindrical capillary [7, 8. Close examination of the SEM images of Marín et al. suggests that the hexagonto-square transition occurs where a new layer appears, whereas the square-to-hexagon transition appears within a single layer.

The idea that kinetics prevents a system from being able to fully explore configuration space is well known for the glass transition, where fast temperature quenches and applied stresses have been studied for decades. By comparison, the analogous idea for colloidal systems is relatively new. A sure lesson of the present work is this: the dynamics of the deposition, rather than the stability of static packing arrangements, determines the patterns that ultimately form [9 11.

Perhaps the final missing piece of the coffee-ring puzzle is a "4D" picture of the deposition at the edge. How does this jittery game of microscopic Tetris actually play out in three dimensions plus time? Is there a fluid layer remaining above the deposit? If so, what is the nature of this layer and does it alter the evaporative and fluid fluxes at the edge? As for the problems that remain, like the coffee stains on Nagel's countertop, it's fair to say that so far no one has them completely licked.

\section{References}

[1] Á. G. Marín, H. Gelderblom, D. Lohse, and J. H. Snoeijer, Phys. Rev. Lett. 107, 085502 (2011).

[2] R. D. Deegan et al., Nature 389, 827 (1997); Phys. Rev. E 62, 756 (2000).

[3] C. J. Curtis et al., in Proceedings of SIGGRAPH '97, (ACM Press/Addison-Wesley, New York, 1997); Cassidy Curtis and Kurt Fleischer (private communication).

(C) 2011 American Physical Society 
[4] A.-M. Cazabat and G. Guena, Soft Matter 6, 2591 (2010).

[5] R. D. Deegan, Phys. Rev. E 61, 475 (2000).

[6] C. Allain and L. Limat, Phys. Rev. Lett. 74, 2981 (1995); L. Pauchard, F. Parisse, and C. Allain, Phys. Rev. E 59, 3737 (1999).

[7] P. Pieranski, L. Strzelecki, and B. Pansu, Phys. Rev. Lett. 50, 900 (1983).

[8] M. Abkarian, J. Nunes, and H. A. Stone, J. Am. Chem. Soc.
126, 5987 (2004).

[9] I. Cohen, T. G. Mason, and D. A. Weitz, Phys. Rev. Lett. 93, 046001 (2004).

[10] G. R. Farrell, K. M. Martini, and N. Menon, Soft Matter 6, 2925 (2010).

[11] L. T. Shereda, R. G. Larson, and M. J. Solomon, Phys. Rev. Lett. 101, 038301 (2008).

\section{About the Author}

\section{Greg Huber}

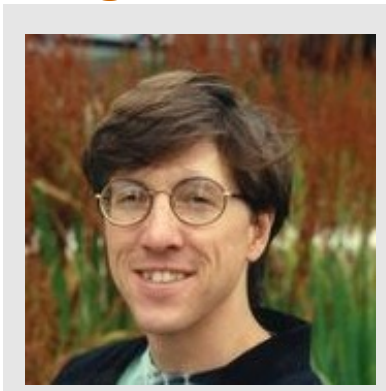

Greg Huber works on problems in biological physics, with an emphasis on the interplay of statistical mechanics, biomechanics, and fluid dynamics. He is an Associate Professor in the Department of Cell Biology at the University of Connecticut Health Center, with joint appointments in the Department of Mathematics and the Department of Physics at the University of Connecticut, Storrs. He received his Ph.D. from Boston University under the supervision of H. Eugene Stanley, working on the statistical physics of order-disorder transitions and turbulence, and his B.S. from MIT, where he worked with Alan Guth on inflation. A post-doctoral appointment at the James Franck Institute of the University of Chicago led him to be one of the first theorists to consider the coffee-ring effect. (Photograph courtesy of George M. Bergman) 